\title{
Thrombus Composition in Sudden Cardiac Death from Acute Myocardial Infarction.
}

Johanne Silvainn ${ }^{1}$, Jean-Philippe Collet ${ }^{1}$, Paul Guedeney ${ }^{1}$, Olivier Varenne ${ }^{2}$, Chandrasekaran Nagaswami ${ }^{3}$, Carole Maupain ${ }^{1}$, Jean-Philippe Empana ${ }^{4}$, Chantal Boulanger ${ }^{4}$, M uriel Tafflet ${ }^{4}$, Stephane M anzo-Silberman ${ }^{5}$, M athieu Kerneis ${ }^{1}$, Delphine Brugier ${ }^{1}$, Nicolas Vignolles ${ }^{1}$, John W. Weisel $^{3}$, Xavier Jouven ${ }^{4}$, Gilles M ontalescot ${ }^{1}$, Christian Spaulding ${ }^{4}$

1 Sorbonne Université - Univ Paris 06 (UPMC), ACTION Study Group, INSERM UM RS 1166, , Institut de Cardiologie, Hôpital Pitié-Sal pêtrière (AP-HP), Paris, France.

2 Cardiology Department, Cochin Hospital, Paris 5 School of M edicine, Rene Descartes University, Paris, France.

3 Department of Cell and Developmental Biology, University of Pennsylvania School of Medicine, Philadelphia, Pennsylvania.

4 Département de cardiologie, Hôpital Européen Georges Pompidou, Université Paris Descartes, Paris Cardiovascular Research Centre (PARCC), INSERM UMRS 970, Paris Sudden Death Expertise Centre, Paris, France.

5 Cardiology department, Inserm U942, Lariboisiere Hospital, Paris Diderot University, Paris, France.

\section{Corresponding author:}

Dr Johanne Silvain, Institut de Cardiologie, Bureau 278, Pitié-Salpêtrière University Hospital, 47 blvd de l'Hôpital, 75013 Paris, France. Tel: +33 01421629 61; Fax: +33 01421629 31. Email: johanne.silvain@aphp.fr

\footnotetext{
Abstract

Background and aim: It was hypothesized that the pattern of coronary occlusion (thrombus composition) might contribute to the onset of ventricular arrhythmia and sudden cardiac death (SCD) in myocardial infarction (MI).

Methods: The TIDE (thrombus and inflammation in sudden death) study included patients with angiographically-proven acute coronary occlusion as the cause of a ST elevation MI (STEM I) complicated by Sudden Cardiac Death (SCD group) or not (STEMI group). Thrombi were obtained by
} 
thrombo-aspiration before primary percutaneous coronary stenting and analyzed with a quantitative method using scanning electron microscopy. We compared the composition of the thrombi responsible for the coronary occlusion between the two groups and evaluated factors influencing its composition.

Results: We included 121 patients and found that thrombus composition was not different between the SCD group ( $n=23$ ) and the STEM I group ( $n=98)$ regarding content of fibrin fibers $(60.3+18.4 \%$ vs. $62.4 \pm 18.4 \%$ respectively, $p=0.68)$, platelets $(16.3 \pm 19.2 \%$ vs. $15.616 .7 \pm \%, p=0.76)$, erythrocytes $(14.6 \pm 12.5 \%$ vs. $13 \pm 12.1 \%, p=0.73)$ and leukocytes $(0.6 \pm 0.9 \%$ vs. $0.8 \pm 1.5 \%, p=0.93)$. Thrombus composition did not differ between patients receiving upstream-use of glycoprotein IIb/ Illa platelet receptor inhibitors (GPI) and patients free of GPI. The only factor found to influence thrombus composition was the ischemic time from symptom onset to primary $\mathrm{PCl}$, with a decreased content in fibrin fibers $(57.8 \pm 18.5 \%$ vs. $71.9 \pm 10.1 \%, p=0.0008)$ and a higher platelet content $(19.2 \pm 19.1 \%$ vs. $7.9 \pm 5.7 \% p=0.014$ ) in early presenters ( $<3$ hours of ischemic time) vs. late presenters ( $>6$ hours of ischemic time).

Conclusion: Composition of intracoronary thrombi in STEMI patients does not differ between those presenting with and without SCD. Time from symptom onset to coronary reperfusion seems to be the strongest factor influencing thrombus composition in MI.

\section{Abbreviation list:}

STEM I: ST-elevated myocardial infarction

SCD: sudden cardiac death

TIDE study: Thrombus and Inflammation in sudden Death study

GPI: glycoprotein Ilb/Illa inhibitors

IQR: interquartile range

Key words: Sudden cardiac death; ST elevated myocardial infarction; coronary thrombus

\section{INTRODUCTION}

Sudden cardiac death (SCD) accounts for 4 million deaths every year worldwide.[1]A frequent cause of SCDs is ventricular fibrillation or fast ventricular tachycardia in the setting of an acute coronary artery occlusion. [2,3]Several risk factors of SCD have been identified in previous studies, however 
the relationship between an acute coronary artery occlusion and the onset of ventricular arrhythmia is unknown.[4-9]

In ST-elevation myocardial infarction (STEMI) patients, a hypothesis is that differences in patterns of STEMI development leading to more rapid coronary occlusion could trigger SCD. In the TIDE (Thrombus and Inflammation in sudden Death) microparticle study, we reported higher concentrations of intracoronary endothelial microparticles in STEMI with SCD at presentation versus those without SCD, suggesting that ventricular arrhythmia in the setting of acute myocardial ischemia is not entirely explained by rhythmic vulnerability.[10] A vascular vulnerability related to a specific pattern of abrupt coronary occlusion may also be involved. The TIDE Thrombus study (NCT00748111) was designed to investigate this hypothesis. Our aim was to assess whether thrombus architecture per se could affect the occurrence of SCD in myocardial infarction and characterize the independent correlates of thrombus composition.

\section{METHODS}

\section{Study design}

We prospectively screened all the STEM I and SCD patients referred to the catheterization laboratory of the Pitié-Salpêtrière and Cochin Hospital in Paris France for primary percutaneous coronary intervention (PCI). In the thrombus analysis of the TIDE study, we included SCD patients with documented STEM I (SCD group) and STEM I patients without ventricular arrhythmias (STEM I group) meeting the following inclusion criteria: age >18 years; documented acute coronary artery occlusion with thrombolysis in myocardial infarction (TIMI) flow of 0,1 , or 2 ; coronary blood sampling using an aspiration catheter available; a time delay from symptom onset to ventricular arrhythmia or pulseless condition of less than $1 \mathrm{~h}$; successful out-of-hospital resuscitation with Return Of Spontaneous Circulation (ROSC) after SCD. Due to logistical constraints, we evaluated only patients presenting during 'on hours'. Our aim was to include 4 STEM I for each SCD. Based on our previous experience in the analysis of thrombus composition in STEM I presenters, we estimated a sample size of 100 STEM I and 25 SCD to be reasonable although no sample size calculation could be performed due to the lack of available data.[11] Recruitment of the TIDE study was slower than expected and patients with STEM I from the thrombus registry of the Pitié-Salpêtrière hospital who matched the inclusion criteria of the TIDE study were included in the present analysis as explained in the figure 1.

\section{Thrombus collection}

Thrombo-aspiration during primary $\mathrm{PCl}$ was performed by a low-profile catheter (Export 6F, M edtronic, Santa Rosa, California). Collected thrombi were immediately washed with saline and fixed with $2 \%$ glutaraldehyde in $50 \mathrm{mmol} / \mathrm{l} \mathrm{Na}$ Cacodylate buffer (pH 7.3). Patients received $250 \mathrm{mg}$ of aspirin intravenously; $600 \mathrm{mg}$ of clopidogrel (crushed and administrated in the nasogastric tube if necessary for SCD patients) and the use of glycoprotein IIb/IIla platelet receptor inhibitors (GPI) were 
administered before and/or during the procedure at the discretion of the physicians.

\section{Study oversight}

The study was reviewed and accepted by the ethics committee of the Institutional Review Board of the Cochin Hospital, Paris, France and the thrombus registry of the Pitié-Salpêtrière is declared as part of the ePARIS STEM I registry which was reviewed and accepted by the ethics committee of the Institutional Review Board of the Pitié-Salpêtrière Hospital. Informed consent was obtained before the procedure in all STEM I patients without SCD. The ethics committee allowed blood sampling and thrombus collection without consent during PCI procedures in case of SCD. However, the data could be used only if informed consent was obtained from the next of kin before or after the procedure and from the patient if $s /$ he survived.

\section{Scanning electron microscope analysis}

Sample fixation, dehydration, and preparation were performed according to a previously published method.[12]High-definition photographs (x3,000 magnification) were obtained using a Philips/FEI XL20 scanning electron microscope 4-nm resolution (FEI, Hillsboro, Oregon). To control for composition heterogeneity in the analysis of the surface of thrombus, we covered several areas (at least 12 according to a grid and the size of the thrombus) for each thrombus and used a validated analysis approach to determine the proportion of each component of thrombus composition (platelet, fibrin, erythrocytes, leukocytes, cholesterol crystal) of images for a single thrombus.[11] Each thrombus analysis comprised for 10 to 15 high magnification scanning electron microscopy (SEM ) pictures at randomly chosen locations to eliminate selection bias. All the structures visualized were easy to identify on the basis of SEM images described previously. SEM and image analysis were performed by two independent trained technicians blinded to the clinical data. Very low interindividual variability was found in the thrombus composition analysis ( $\varangle \%)$.

\section{Microparticle analysis}

Specific microparticle sub-populations were identified by flow cytometry, as previously reported in our previous publication [10]. They were measured in the blood from the culprit coronary lesion, the systemic blood before the coronary intervention (Pre-PCl) and in the systemic blood after the coronary intervention (Post $\mathrm{PCl}$ ). We report in this manuscript the micropaticle results in patients having both thrombus and micropaticle data available within the TIDE study (subanalysis). AnnexinV+microparticles, endothelium-derived microparticles (EM Ps; CD144+), platelet-derived microparticles (CD41+), leukocyte-derived microparticles (CD11at), erythrocyte-derived microparticles (CD235at) were expressed as $\mathrm{nb} / \mu \mathrm{l}$ and compared with two-by-two comparisons of median (interquartile range).

\section{Clinical data collection}

Clinical data were prospectively collected at admission and during the hospital stay. A certified core laboratory reviewed all angiographic data. Clinical and biological data were analyzed to identify 
predictors of thrombus composition. For SCD patients, the location of SCD, initial cardiac rhythm, intervals between the onset of SCD and basic life support ('no-flow'), between basic life support and ROSC ('low-flow') were collected from the emergency medical system physicians according to the Utstein recommendations. [13]

\section{Statistical analyses}

Descriptive statistics are reported as mean $\pm S D$, median and interquartile range (IQR) $\left(25^{\text {th }}\right.$ to $\left.75^{\text {th }}\right)$ percentiles and percentages when appropriate. Differences in baseline characteristics between two groups were compared using Chi Square (Fisher exact test if expected count «5). Potential associations between clinical and biological parameters such as thrombus composition were compared by Student's t-test and ANOVA test. As performed in a previous publication we divided the data in 3 groups on the basis of ischemic time and we used patients with ischemic time < 3 hours as the reference group.[11] A Kruskal-Wallis test was used for the trend between the three groups. Statistical analysis was performed with Graphpad Prism (GraphPad Software, Inc., USA).

\section{RESULTS}

Between March 2007 and December 2009, 77 patients were prospectively recruited in the TIDE study, and 44 patients of the Pitié-Salpêtrière Thrombus registry matching the inclusion criteria of the TIDE study were added to complete the final analysis (Figure 1). Of the 121 STEM I patients with thrombus analyzed, 23 were in the SCD group and 98 in the STEMI group. Patients in the STEM I group had longer median ischemic times than patients included in the SCD group (120 min [IQR at $60-179$ ]) vs. 207min [IQR at 145-325]), $p=0.0008$ ) (Table 1). The rate of glycoprotein Ilb/Illa inhibitors (GPI) use was higher in the STEM I group than the SCD group due to upstream prehospital use. In the SCD group, the first recorded rhythm was ventricular tachycardia or ventricular fibrillation in the vast majority of patients (Table 2). The median values before any resuscitation (no-flow) was 0 min (IQR $0-5$ ) reflecting a group of SCD patients with immediate or fast care, and the median values before the return of spontaneous circulation (low-flow) was $18.5 \mathrm{~min}$ (IQR 10-26). As expected, in-hospital mortality rate was much lower in the STEM I group as compared with the SCD group.

\section{Thrombus analysis}

Fibrin fibers were the major component of the thrombus representing more than $60 \%$ of its composition. Platelets, erythrocytes, cholesterol crystals and leukocytes all together represented the remaining $40 \%$ (Figure 2). Components of thrombus could be easily identified in our SEM high definition pictures (supplemental figure S1). Examples of the 1210 SEM pictures analysed in this study are shown in supplemental figure S2A with an analysis of a thrombus from the SCD group and in supplemental figure S2B with an analysis of a thrombus from the STEM I group. We did not find any difference in thrombus composition between the two groups of patients (Table 3).

\section{Impact of GPI and ischemic time on thrombus composition}

GPI was administered in the prehospital setting to one third of the patients. There was no difference 
in fibrin content (respectively $61.0 \pm 21.6 \%, 63.6 \pm 16.4 \%$ and $61.3 \pm 18.7 \%, p=0.897$ ) and platelet content (respectively $12.1+13.4 \%, 12.5+13.9 \%$ and $18.1+19.1 \%, p=0.197$ ) of the thrombus between the GPI-free group, upstream-use and per-procedural-use of GPI groups, respectively. It is noteworthy that ischemic time did not differ according to GPI use (median of 210min [IQR at 80-360], 185min [IQR at 125-245] and 175min [IQR at 132-356] for GPI-free, upstream-use and perprocedural-use respectively, $p=0.83$ ).

The total ischemic time, defined as the time delay from symptom onset to thrombus retrieval, was the only factor impacting thrombus composition. Platelet content decreased from $9.2 \pm 19.1 \%$ to $7.9 \pm 5.1 \%$ among patients with an ischemic time of $<3$ hours and $>6$ h, respectively ( $p=0.014$ for trend) while fibrin content increased from $57.8+18.5 \%$ to $71.9 \pm 10.1 \%$ ( $p=0.0008$ for trend) (igure $\mathbf{3}$ ). Content in red cells and white cells was not affected by ischemic time.

\section{Microparticle analysis}

We presented in the supplemental table $\mathbf{S 1}$ the results of the sub-analysis of microparticle measurement in the SCD and STEM I groups, in patients having both thrombus and microparticle data available. Data were consistent with our previous finding with higher concentration of M P in the SCD group, especially the platelet-derived microparticles (CD41+) in the systemic blood prePCl.

\section{DISCUSSION}

The TIDE thrombus study was designed to demonstrate whether the pattern of coronary occlusion (thrombus composition) could contribute to the onset of ventricular arrhythmia and SCD in myocardial infarction and to characterize the independent correlates of thrombus composition. The main results of our study can be summarized as follows. First, intra-coronary thrombi retrieved during percutaneous myocardial revascularization are mainly made of fibrin. Second, there is no difference in thrombus composition between STEM I presenters complicated by SCD and STEM I without SCD. Third, the use of GPI had no impact on thrombus composition even with early prehospital treatment initiation. Fourth, ischemic time is the only independent predictor of the thrombus content.

Manual thrombectomy was a common adjunctive technique to $\mathrm{PCI}$ in STEMI patients, allowing better coronary stent implantation and a lower rate of stent thrombosis, until randomized trials demonstrated no mortality benefit and an increased risk of stroke. [14-16] This device offers a unique opportunity to study intracoronary thrombus in STEM I patients using various approaches, histopathology, immunohistochemistry, angioscopy or scanning electron microscopy. [11,17-19] The results of the TIDE study are in line with our previous findings on thrombus composition in myocardial infarction and confirm the crucial role of fibrin fibers as the main component of the architecture of intra-coronary thrombus, whereas platelets and erythrocytes are second in line in 
terms of composition.[11] We also found that there was a large variation in the proportion of each component of thrombus composition, with percentages of fiber or platelet content in a thrombus both varying from $0 \%$ to $80 \%$, indicating a large variation in terms of thrombus composition in an individual patient. Some thrombi are mainly composed of platelets and some mainly of fibrin fibers.

TIDE also demonstrates that ischemic time is the main factor influencing thrombus composition, with a decrease of platelet content and an increase in fibrin content over time. This is also in line with our previous work, although confirmed here in a much larger sample of patients.[11] These results are consistent with recent studies from other groups and with the generally accepted pathogenesis of clot formation where platelets trigger thrombus formation and are more present in "fresh" thrombi, while fibrin strengthens the overall clot structure and comes into play later on.[2022] The absence of difference in thrombus composition according to the use of GPI was unexpected and cannot be interpreted outside the results of previous randomized trials showing a benefit in early GPI administration in the prehospital setting.[23]

The hypothesis that STEM I patients with ventricular arrhythmia leading to SCD have a different pattern of coronary thrombus composition (more platelet-rich) as compared to STEM I patients without SCD was not confirmed by the present work. Indeed, thrombus architecture analyzed in both groups were similar despite the fact that SCD patients had reduced ischemic time and less frequent upstream use of GPI as compared to STEMI patients without cardiac arrest. In the TIDE inflammatory study published previously, intracoronary concentration of endothelial microparticles (EM P) were significantly higher among SCD patients compared to STEM I patients supporting the hypothesis that occurrence of fast ventricular tachycardia or ventricular fibrillation in the setting of myocardial infarction is not explained only by a rhythmic vulnerability but could also involve a vascular vulnerability.[10] In the present work, we did not manage to find any specific pattern with SCD, as we found no significant difference between our two populations. Although we found differences in platelet microparticles (CD41+) level which were increased in the SCD group, and especially in the systemic blood samples measured before the coronary intervention, our results were most likely affected by the difference in the upstream use of GPI which was higher in the STEM I group.

We acknowledged several limitations. First, STEMI patients screened in this trial were selected and might not be representative of the entire population. Second, the analyses were performed on the main aspirated piece of thrombus and we may have missed the most informative pieces, which may have been damaged during the process of aspiration. This may account for the lack of effect of GPI on thrombus composition. However, the number of thrombi retrieved and the random localization of the pictures that were analyzed should guarantee an unbiased measurement and control for composition heterogeneity in the pieces of thrombi that were obtained. In addition, intra-coronary thrombi are stiff structures and much more stable than in vitro clots, and the 
preserved appearance of cells and fibrin fibers in the microscopy images confirmed that distortion was minimal. [24] Third, although the fixation of thrombus sample in the glutaraldehyde solution gave the advantages to be conserved almost eternally for additional imaging analysis, such method does not allow additional immunological analysis and may limit the extent of the comprehension of this analysis. Finally, the aspirated thrombus may be older than expected from the duration of the ischemic time and younger thrombus could be superimposed with an older thrombus, thereby potentially confounding our observations.

\section{CONCLUSION}

The composition of intracoronary thrombi that is responsible for acute myocardial infarction does not differ according to the occurrence of SCD at presentation. Ischemic time remains the main factor impacting thrombus composition in patients with myocardial infarction.

\section{Conflicts of interest}

Johanne Silvain reports the following disclosures during the past 2 years : Research Grants to Institution from the Fondation de France and the Institute of Cardiolometabolism (ICAN); Consulting fees from Actelion, Amed, Astra-Zeneca, Bayer, Daiichi-Sankyo, Eli Lilly, Gilead Science and SanofiAventis; Speaker honorariums from AstraZeneca, Amgen, Algorythm , Daiichi Sankyo, Eli Lilly, Iroko Cardio and Travels Support from Amgen , Astra-Zeneca and Saint-Jude M edical.

Jean-Philippe Collet reports the following disclosures: Research Grants to Institution or honorarium from AstraZeneca, Bayer, Bristol-M yers Squibb, Daiichi-Sankyo, Eli-Lilly, Fédération Française de Cardiologie, Lead-Up, M edtronic, M SD, Sanofi-Aventis, WebMD.

Paul Guedeney does not report any conflict of interest.

Olivier Varenne does not report any conflict of interest.

Chandrasekaran Nagaswami does not report any conflict of interest.

Carole Maupain does not report any conflict of interest.

Jean-Philippe Empana does not report any conflict of interest.

Chantal Boulanger does not report any conflict of interest.

M uriel Tafflet does not report any conflict of interest.

Stephane M anzo-Silberman does not report any conflict of interest.

Delphine Brugier does not report any conflict of interest.

Nicolas Vignolles does not report any conflict of interest.

Xavier Jouven does not report any conflict of interest.

John W. Weisel reports the following disclosures during the past 2 years: Research grant to Institution from Bayer Healthcare; expert witness for Jenner and Block LLP. 
Gilles Montalescot reports the following disclosures: Research Grants to Institution or honorarium from ADIR, Amgen, AstraZeneca, Bayer, Berlin Chimie AG, Boehringer Ingelheim, Bristol-M yers Squibb, Beth Israel Deaconess M edical, Brigham Women's Hospital, Cardiovascular Research Foundation, Celladon, CME Resources, Daiichi-Sankyo, Eli-Lilly, Europa, Elsevier, Fédération Française de Cardiologie, Fondazione Anna Maria Sechi per il Cuore, Gilead, ICAN, Janssen, Lead-Up, M enarini, M edtronic, MSD, Pfizer, Sanofi-Aventis, Servier, The M edicines Company, TIMI Study Group, WebMD.

Christian Spaulding reports the following disclosures in the past two years: research grants from the French M inistry of Health, consulting fees from Abiomed, Zoll, M edtronic, M edpass, speaker fees from Astra-Zeneca, Cordis, Servier, Lead-Up, Bayer, the M edicines Company, Eli Lilly, WebMD.

Funding:

ANR, ACTION study group www.actioncoeur.fr

\section{References}

[1] Priori SG, Blomström-Lundqvist C, M azzanti A, et al. 2015 ESC Guidelines for the management of patients with ventricular arrhythmias and the prevention of sudden cardiac death: The Task Force for the M anagement of Patients with Ventricular Arrhythmias and the Prevention of Sudden Cardiac Death of the European Society of Cardiology (ESC). Endorsed by: Association for European Paediatric and Congenital Cardiology (AEPC). Eur Heart J 2015;36:2793-867. doi:10.1093/eurheartj/ehv316.

[2] Spaulding CM, Joly LM, Rosenberg A, et al. Immediate coronary angiography in survivors of out-of-hospital cardiac arrest. N Engl J Med 1997;336:1629-33. doi:10.1056/ NEJM 199706053362302.

[3] Eisenberg MS, M engert TJ. Cardiac resuscitation. N Engl J M ed 2001;344:1304- 13. doi:10.1056/ NEJ M 200104263441707.

[4] Siscovick DS, Weiss NS, Fletcher RH, Lasky T. The incidence of primary cardiac arrest during vigorous exercise. N Engl J Med 1984;311:874-7. doi:10.1056/ NEJ M 198410043111402.

[5] Jouven X, Desnos M, Guerot C, Ducimetière P. Predicting sudden death in the population: the Paris Prospective Study I. Circulation 1999;99:1978-83.

[6] Albert CM, Chae CU, Grodstein F, et al. Prospective study of sudden cardiac death among women in the United States. Circulation 2003;107:2096-101. doi:10.1161/01.CIR.0000065223.21530.11. 
[7] Albert CM, Oh K, Whang W, M anson JE, et al. Dietary alpha-linolenic acid intake and risk of sudden cardiac death and coronary heart disease. Circulation 2005;112:3232-8. doi:10.1161/CIRCULATIONAHA.105.572008.

[8] Jouven X, Empana J-P, Schwartz PJ, Desnos M, Courbon D, Ducimetière P. Heart-rate profile during exercise as a predictor of sudden death. N Engl J M ed 2005;352:1951-8. doi:10.1056/ NEJM oa043012.

[9] Dekker LRC, Bezzina CR, Henriques JPS, et al. Familial sudden death is an important risk factor for primary ventricular fibrillation: a case-control study in acute myocardial infarction patients. Circulation 2006;114:1140-5. doi:10.1161/CIRCULATIONAHA.105.606145.

[10] Empana J-P, Boulanger CM , Tafflet M, et al. Microparticles and sudden cardiac death due to coronary occlusion. The TIDE (Thrombus and Inflammation in sudden DEath) study. Eur Heart J Acute Cardiovasc Care 2015;4:28-36. doi:10.1177/2048872614538404.

[11] Silvain J, Collet J-P, Nagaswami C, et al. Composition of coronary thrombus in acute myocardial infarction. J Am Coll Cardiol 2011;57:1359-67. doi:10.1016/j.jacc.2010.09.077.

[12] Weisel JW, Nagaswami C. Computer modeling of fibrin polymerization kinetics correlated with electron microscope and turbidity observations: clot structure and assembly are kinetically controlled. Biophys J 1992;63:111-28. doi:10.1016/S00063495(92)81594-1.

[13] Perkins GD, Jacobs IG, Nadkarni VM , et al. Cardiac arrest and cardiopulmonary resuscitation outcome reports: update of the Utstein Resuscitation Registry Templates for Out-of-Hospital Cardiac Arrest: a statement for healthcare professionals from a task force of the International Liaison Committee on Resuscitation (American Heart Association, European Resuscitation Council, Australian and New Zealand Council on Resuscitation, Heart and Stroke Foundation of Canada, InterAmerican Heart Foundation, Resuscitation Council of Southern Africa, Resuscitation Council of Asia); and the American Heart Association Emergency Cardiovascular Care Committee and the Council on Cardiopulmonary, Critical Care, Perioperative and Resuscitation. Circulation 2015;132:1286-300. doi:10.1161/CIR.0000000000000144.

[14] Windecker S, Kolh P, Alfonso F, et al. 2014 ESC/EACTS Guidelines on myocardial revascularization: The Task Force on M yocardial Revascularization of the European 
Society of Cardiology (ESC) and the European Association for Cardio-Thoracic Surgery (EACTS)Developed with the special contribution of the European Association of Percutaneous Cardiovascular Interventions (EAPCI). Eur Heart J 2014;35:2541-619. doi:10.1093/eurheartj/ehu278.

[15] Fröbert O, Lagerqvist B, Olivecrona GK, et al. Thrombus aspiration during ST-segment elevation myocardial infarction. N Engl J Med 2013;369:1587-97. doi:10.1056/ NEJ M oa1308789.

[16] Jolly SS, Cairns JA, Yusuf S, et al. Randomized trial of primary PCI with or without routine manual thrombectomy. N Engl J Med 2015;372:1389-98. doi:10.1056/ NEJ M oa1415098.

[17] Sato Y, Hatakeyama K, Yamashita A, M arutsuka K, Sumiyoshi A, Asada Y. Proportion of fibrin and platelets differs in thrombi on ruptured and eroded coronary atherosclerotic plaques in humans. Heart Br Card Soc 2005;91:526-30. doi:10.1136/ hrt.2004.034058.

[18] Kramer M CA, van der Wal AC, Koch KT, et al. Presence of older thrombus is an independent predictor of long-term mortality in patients with ST-elevation myocardial infarction treated with thrombus aspiration during primary percutaneous coronary intervention. Circulation 2008;118:1810-6. doi:10.1161/CIRCULATIONAHA.108.780734.

[19] Uchida Y, Uchida Y, Sakurai T, Kanai M, Shirai S, M orita T. Characterization of coronary fibrin thrombus in patients with acute coronary syndrome using dye-staining angioscopy. Arterioscler Thromb Vasc Biol 2011;31:1452-60. doi:10.1161/ATVBAHA.110.221671.

[20] Iwata H, Sata M, Ando J, et al. Impact of primitive cells in intracoronary thrombi on lesion prognosis: temporal analysis of cellular constituents of thrombotic material obtained from patients with acute coronary syndrome. Heart Br Card Soc 2010;96:74855. doi:10.1136/hrt.2009.181040.

[21] Zalewski ], Bogaert J, Sadowski M , et al. Plasma fibrin clot phenotype independently affects intracoronary thrombus ultrastructure in patients with acute myocardial infarction. Thromb Haemost 2015;113:1258-69. doi:10.1160/TH14-09-0801.

[22] Furie B, Furie BC. M echanisms of thrombus formation. N Engl J M ed 2008;359:938-49. doi:10.1056/ NEJ M ra0801082.

[23] Van't Hof AWJ, Ten Berg J, Heestermans T, et al. Prehospital initiation of tirofiban in patients with ST-elevation myocardial infarction undergoing primary angioplasty (On- 
TIME 2): a multicentre, double-blind, randomised controlled trial. Lancet Lond Engl 2008;372:537-46. doi:10.1016/50140-6736(08)61235-0.

[24] Collet J-P, Shuman H, Ledger RE, Lee S, Weisel JW. The elasticity of an individual fibrin fiber in a clot. Proc Natl Acad Sci U S A 2005;102:9133-7. doi:10.1073/ pnas. 0504120102. 


\section{Figure 1: Flow chart of the study}

STEMI: ST-segment elevated myocardial infarction; SCD: sudden cardiac death; PCI: percutaneous coronary intervention

Figure 2: Thrombus composition (percentages of each element) in the study population of $n=121$ Thrombus (Sudden cardiac death group on the right and STEMI group on the left). Red Lines indicate median and interquartile range (25\% to $75 \%)$.

Figure 3: Relation between ischemic time (duration of thrombus formation) and thrombus composition. P-values are given for comparison with the group $<3$ hours as a reference with multiple student t-test. * $p$ value $\varangle .05$. The Kruskal Wallis test for comparison of the three groups resulted in $p$ value $=0.048$ for the fibrin fibers and $p=0.047$ for platelets.

\section{Figure 1:}




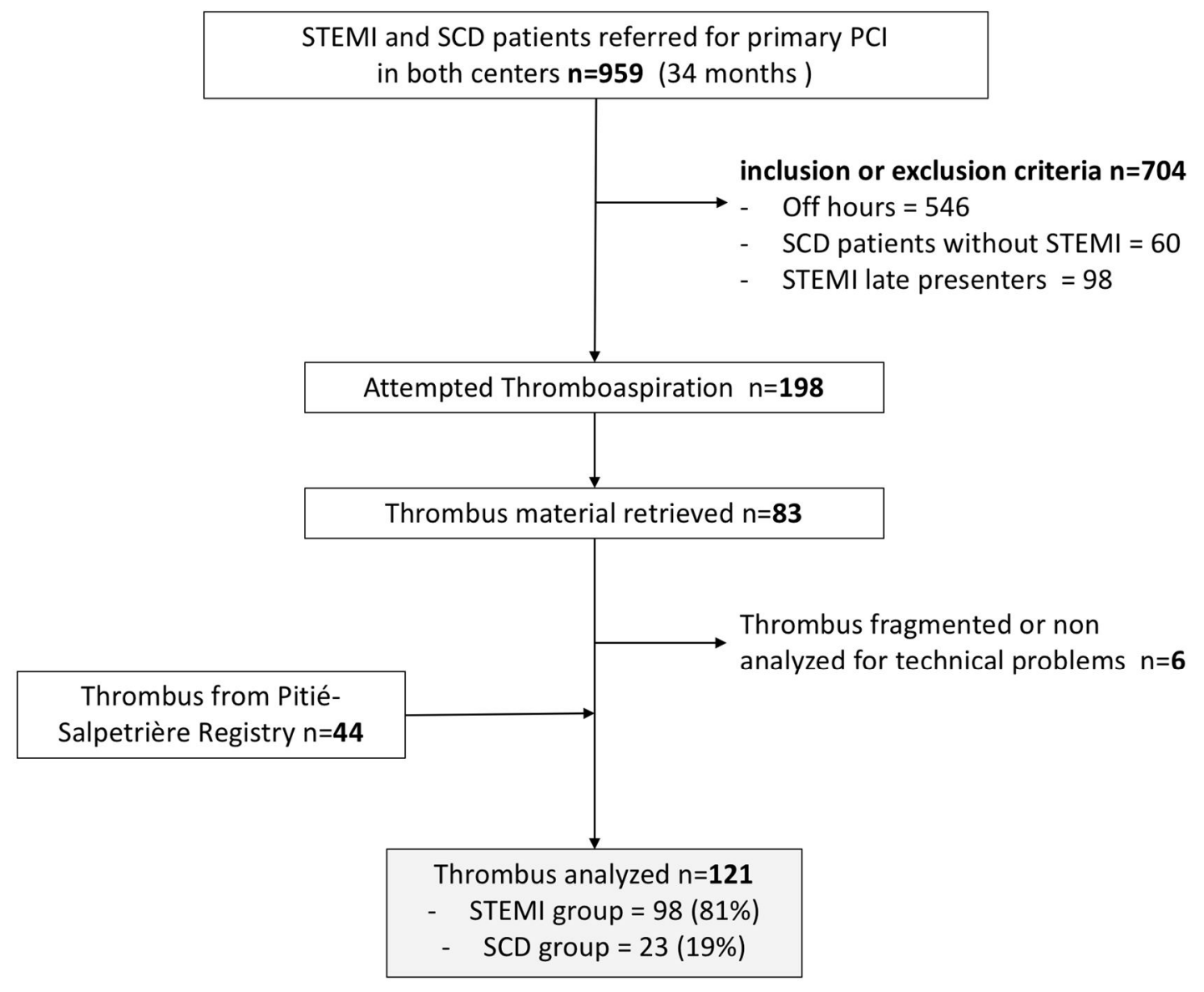




\section{Figure 2:}

Uncomplicated ST-Elevation MI

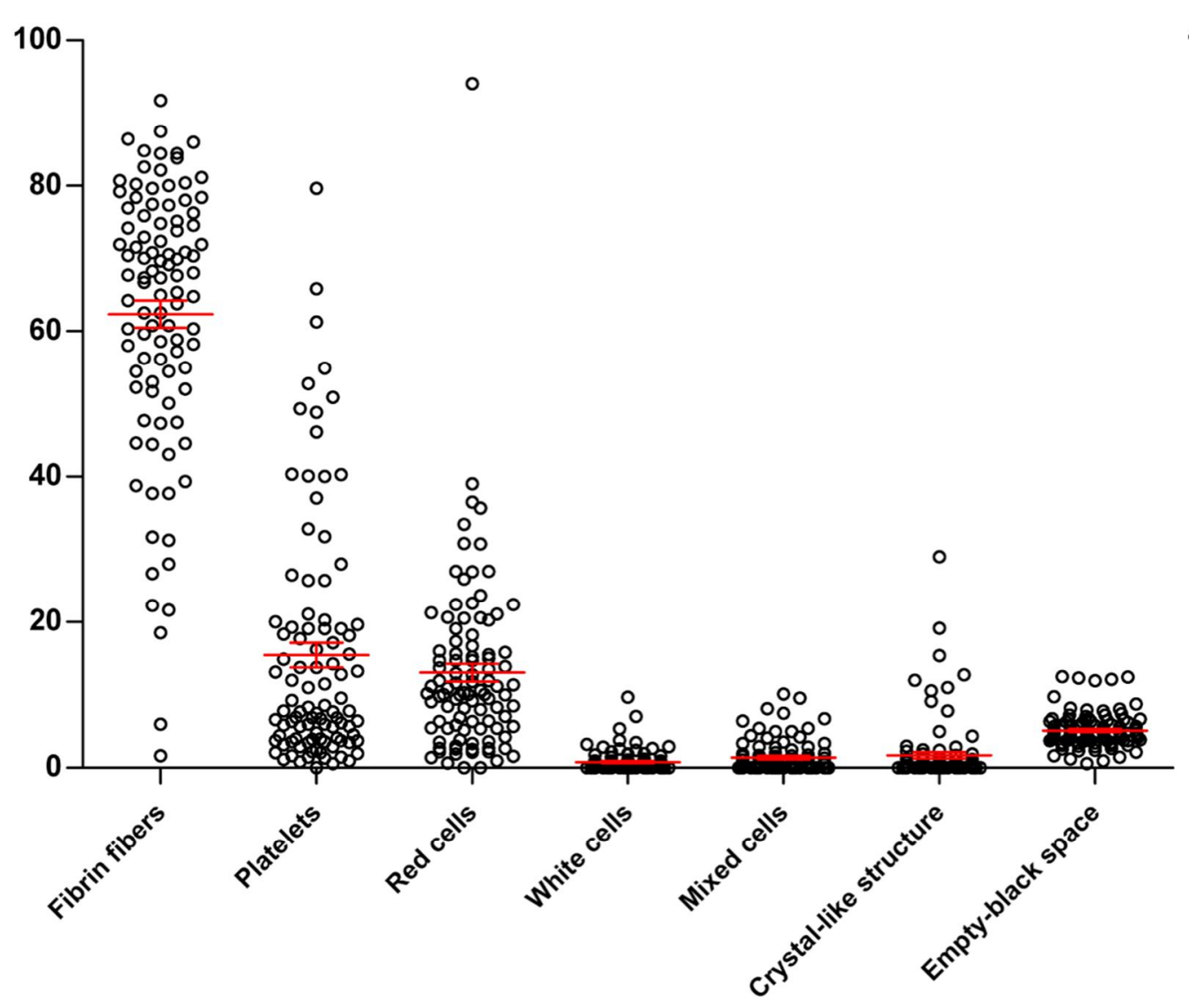

ST-Elevation MI with Sudden Cardiac Death

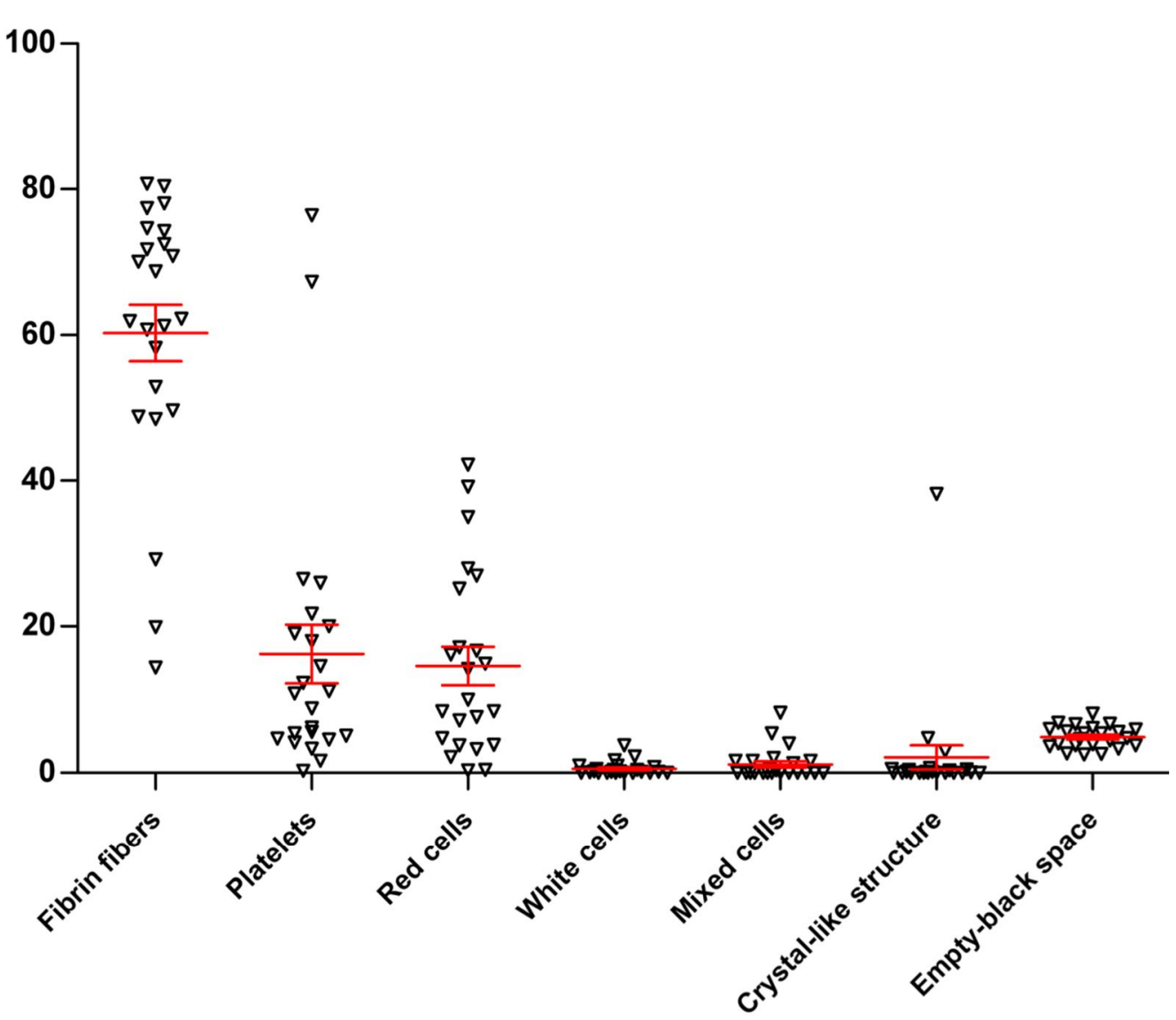


Figure 3:

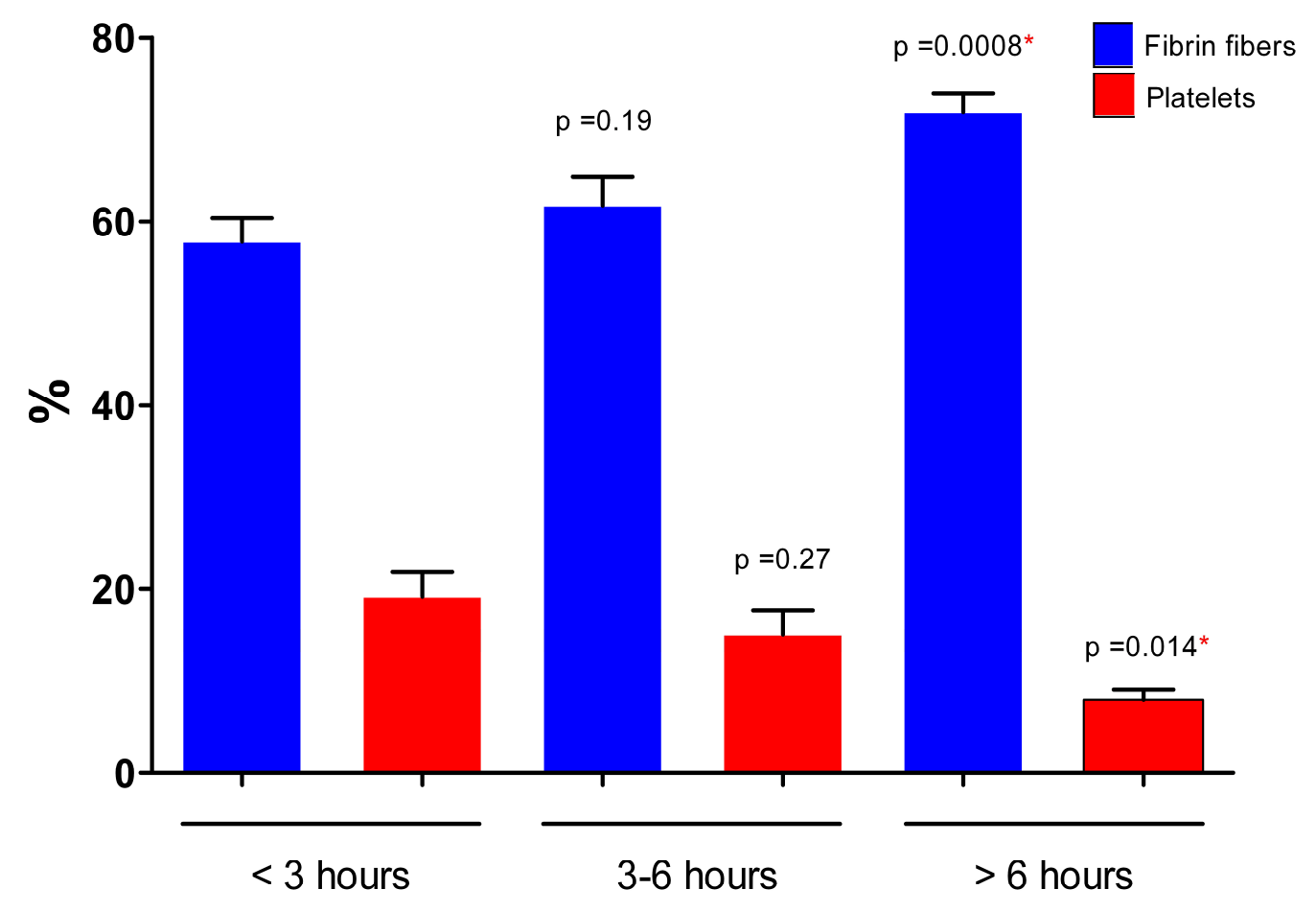


Table 1: Demographic and procedural characteristics description. * indicates a significant results with a $p$ value $<0.05$. Values are mean $\pm S D, n(\%)$ or median [interquartile range].

\begin{tabular}{|c|c|c|c|}
\hline & $\begin{array}{l}\text { Sudden Cardiac } \\
\text { Death group }(n=23)\end{array}$ & $\begin{array}{l}\text { STEMI group } \\
(n=98)\end{array}$ & p value \\
\hline \multicolumn{4}{|l|}{ Demographic and CV risk factors } \\
\hline Age (years) & $58.6 \pm 13.4$ & $57.9 \pm 13$ & 0.83 \\
\hline Female gender & $2(8.7 \%)$ & $18(18.3 \%)$ & 0.36 \\
\hline Body M ass Index & $25.1 \pm 4.3$ & $26.5 \pm 4.4$ & 0.49 \\
\hline Known Hypertension & $11(47.8 \%)$ & $33(33.6 \%)$ & $0.024 *$ \\
\hline Diabetes & $4(17.4 \%)$ & $15(15.3 \%)$ & 0.75 \\
\hline Dyslipidemia & $7(30.4 \%)$ & $40(40.8 \%)$ & 0.36 \\
\hline Family History of Coronary Disease & $4(17.4 \%)$ & $30(30.6 \%)$ & 0.3 \\
\hline Active smoking & $9(39.1 \%)$ & $43(43.9 \%)$ & 0.68 \\
\hline \multicolumn{4}{|l|}{ Past M edical History } \\
\hline Ischemic heart disease & $5(21.7 \%)$ & $11(11.2 \%)$ & 0.18 \\
\hline Coronary revascularization & $5(21.7 \%)$ & $10(10.2 \%)$ & 0.18 \\
\hline Stroke & $0(0 \%)$ & $3(5.1 \%)$ & 1 \\
\hline Chronic Aspirin treatment & $3(13 \%)$ & $12(12.2 \%)$ & 1 \\
\hline Chronic Clopidogrel treatment & $3(13 \%)$ & $3(3.1 \%)$ & 0.082 \\
\hline \multicolumn{4}{|l|}{ Procedural Characteristics } \\
\hline Ischemic Time in min (M edian - IQR) & $120[60-179]$ & $207[145-325]$ & $0.001^{*}$ \\
\hline \multicolumn{4}{|l|}{ Extension of the Coronary artery disease } \\
\hline Single-vessel lesion & $15(65.3 \%)$ & $66(67.3 \%)$ & 0.84 \\
\hline Two-vessel lesion & $3(13 \%)$ & $25(25.5 \%)$ & 0.18 \\
\hline Multi-vessel lesion & $5(21.7 \%)$ & $7(7.1 \%)$ & $0.035^{*}$ \\
\hline \multicolumn{4}{|l|}{ Culprit lesion } \\
\hline Left anterior descending artery & $11(47.8 \%)$ & $51(52 \%)$ & 0.72 \\
\hline Circumflex artery & $4(17.4 \%)$ & $9(9.2 \%)$ & 0.28 \\
\hline Right coronary artery & $8(34.8 \%)$ & $38(38.8 \%)$ & 0.81 \\
\hline \multicolumn{4}{|l|}{ TIMI Flow on initial angiogram } \\
\hline TIMI 0 & $19(82.6 \%)$ & $80(82.5 \%)$ & 0.14 \\
\hline TIMI 1 & $1(4.4 \%)$ & $6(6.2 \%)$ & 1 \\
\hline TIMI 2 & $3(13 \%)$ & $4(4.1 \%)$ & 0.08 \\
\hline TIMI 3 & 0 & $7(7.2 \%)$ & 0.6 \\
\hline $\mathrm{PCl}$ attempted & $21(91.3 \%)$ & $91(93.8 \%)$ & 1 \\
\hline
\end{tabular}




\begin{tabular}{|c|c|c|c|}
\hline Successful PCl & $21(100 \%)$ & $86(92.8 \%)$ & 0.58 \\
\hline Number of stent implanted & $1.2 \pm 0.7$ & $1.3 \pm 0.6$ & 0.55 \\
\hline$\%$ of Drug eluted stent & $2(9.5 \%)$ & $15(16.8 \%)$ & 0.52 \\
\hline Overall Stent Length (mm) & $24.1 \pm 14.1$ & $25.5 \pm 10.9$ & 0.28 \\
\hline Maximal length stent (mm) & $3 \pm 0.43$ & $3.8 \pm 3.7$ & 0.068 \\
\hline Use of Gpllbllla inhibitors & $15(65.2 \%)$ & $89(90.8 \%)$ & $0.0015^{*}$ \\
\hline - Upstream use & $2(8.7 \%)$ & $34(34.7 \%)$ & $0.02 *$ \\
\hline - Cath lab use & $13(56.5 \%)$ & $55(56.1 \%)$ & 0.97 \\
\hline \multicolumn{4}{|l|}{ Post- PCl } \\
\hline Cardiogenic shock & $15(65.3 \%)$ & $10(10.2 \%)$ & $\varangle 0.001^{*}$ \\
\hline Intra-aortic balloon pump use & $9(47.4 \%)$ & $1(1 \%)$ & $\varangle 0.001 *$ \\
\hline Ventricular assistance & $3(15.8 \%)$ & $2(2 \%)$ & $0.02 *$ \\
\hline Ejection Fraction (\%) & $40.7 \pm 15.2$ & $49 \pm 11.7$ & $0.042^{*}$ \\
\hline Peak of Troponin $(\mu \mathrm{g} / \mathrm{L})$ & $60.7 \pm 102.8$ & $96.3 \pm 100.8$ & $0.05^{*}$ \\
\hline Peak of Creatine Phosphokinase (mmol/L) & $3826 \pm 3588$ & $2916 \pm 2412$ & 0.62 \\
\hline
\end{tabular}


Table 2: Specificity of Sudden Cardiac Death Patients

Values are $n(\%)$, median [interquartile range] or mean \pm SD.

\begin{tabular}{|l|l|}
\hline & $\begin{array}{l}\text { Sudden Cardiac Death } \\
\text { group (n=23) }\end{array}$ \\
\hline Ventricular Tachycardia or Ventricular Fibrillation & $18(78.3 \%)$ \\
\hline Asystole & $5(21.7 \%)$ \\
\hline No Flow (min) & $0[0-5]$ \\
\hline Low Flow (min) & $18.5[10-25.7]$ \\
\hline Adrenaline max (mg) & $4 \pm 4$ \\
\hline
\end{tabular}


Table 3: Thrombus composition according to clinical presentation

Values are mean \pm SD. STEMI: ST-segment elevated myocardial infarction.

\begin{tabular}{|l|l|l|l|l|}
\hline & $\begin{array}{l}\text { All patients } \\
\mathbf{( n = 1 2 1 )}\end{array}$ & $\begin{array}{l}\text { Sudden Cardiac } \\
\text { Death }(\mathbf{n = 2 3 )}\end{array}$ & $\begin{array}{l}\text { Uncomplicated } \\
\text { STEMI (n=98) }\end{array}$ & p value \\
\hline Fibrin (\%) & $61.8 \pm 18.4$ & $60.3 \pm 18.5$ & $62.4 \pm 18.4$ & 0.68 \\
\hline Platelet (\%) & $15.6 \pm 17.1$ & $16.3 \pm 19.2$ & $15.6 \pm 16.7$ & 0.76 \\
\hline Red cells (\%) & $13.5 \pm 12.2$ & $14.6 \pm 12.5$ & $13.0 \pm 12.1$ & 0.73 \\
\hline White cells (\%) & $0.8 \pm 1.4$ & $0.6 \pm 0.9$ & $0.8 \pm 1.5$ & 0.93 \\
\hline Mixed Cell-fibrin (\%) & $1.4 \pm 2.2$ & $1.2 \pm 2.1$ & $1.4 \pm 2.2$ & 0.47 \\
\hline Crystal-like structure & $1.8 \pm 5.3$ & $2.1 \pm 7.9$ & $1.7 \pm 4.5$ & 0.87 \\
\hline Empty blank space (\%) & $5.1 \pm 2.2$ & $4.9 \pm 1.5$ & $5.1 \pm 2.4$ & 0.96 \\
\hline
\end{tabular}

